\title{
Tear meniscus evaluation after microkeratome laser in situ keratomileusis, femtosecond laser and femtosmile laser techniques using anterior segment optical coherence tomography
}

This article was published in the following Dove Press journal:

Clinical Ophthalmology

Yasmine Maher Shaaban

Tamer Abdel Fattah Badran

Faculty of Medicine, Ain Shams

University, Cairo, Egypt
Correspondence: Yasmine Maher Shaaban

49 Mohamed Farid Street, Heliopolis,

I I35 I Cairo, Egypt

Tel +20 I2 8893 I888

Email yasminemaher0@gmail.com
Purpose: The purpose of this study was to compare the effects of three different corneal refractive surgeries: microkeratome laser in situ keratomileusis (LASIK), femtosecond laser in situ keratomileusis (FS-LASIK) and femtosecond small incision lenticule extraction (FS-SMILE) on 6-month postoperative stability of tear film to provide a basis for selection of operative procedures.

Patients and methods: This is a prospective, randomized, comparative study that included 90 eyes of three equal groups of patients. Each group was subjected to a different laser technique: LASIK, FS-LASIK and FS-SMILE. Using anterior segment spectral domain optical coherence tomography (AS-SD-OCT), the lower tear meniscus parameters were measured preoperatively and 1 week, 1 month, 3 months and 6 months postoperatively. Changes were studied and compared.

Results: There was a statistically significant difference in tear meniscus height (TMH) between FS-SMILE and FS-LASIK in the first week $(P=0.003)$ and first month $(P=0.002)$ with no statistically significant difference between both techniques at 3 and 6 months postoperatively. In FS-SMILE, TMH returned to $95 \%$ of its preoperative level after 1 month. There was no statistically significant difference in TMH between FS-LASIK and microkeratome LASIK techniques after 1 week and 1 month, but there was a statistically significant difference between both techniques in 3 months $(P=0.019)$ and 6 months $(P=0.032)$. Tear meniscus area (TMA) showed no statistically significant difference between FS-SMILE and FS-LASIK at all points of follow-up, but there was a statistically significant difference between both techniques and microkeratome LASIK (all $P<0.05$ ). Microkeratome LASIK parameters failed to reach the preoperative level till 6 months.

Conclusion: Early recovery of the lower TMH can be achieved as early as 1 month postoperatively with the FS-SMILE technique compared to FS-LASIK and microkeratome LASIK techniques.

Keywords: LASIK, tear meniscus, optical coherence tomography

\section{Introduction}

Refractive surgery is one of the commonest cosmetic procedures performed all over the world. Many treatment options are available, and different procedures have been used. These include the conventional laser in situ keratomileusis (LASIK) in which a microkeratome is used to make a surgical flap followed by stromal ablation, femtosecond laser in situ keratomileusis (FS-LASIK) in which pulses of short duration are 
used to create a thin-hinged corneal flap without using any blade followed by stromal ablation and femtosecond small incision lenticule extraction (FS-SMILE) in which there is no flap and only small laser incision to extract the lenticule (stromal tissue removal) and stromal ablation have been replaced with refractive lenticule removal. ${ }^{1,2}$

Most people who undergo these procedures may encounter at least some dryness, which may be temporary and mild or severe and permanent. The etiology for this postoperative dry eye is not known, but ocular surface disruption during the procedure is commonly considered to be related to the development of dry eye. The most commonly accepted theories suggest the following: 1) the interruption in the corneal nerve fibers at the time of flap creation, followed by decrease in corneal sensitivity, decrease in blink rate and rise in the tear film evaporation by tissue ablation; 2) when a microkeratome is used, it causes a pressure on the cornea during flap creation, which may harm conjunctiva goblet cells leading to an unstable tear mucin layer. This may cause the tears to evaporate fast and leave the exposed cornea dry; 3) the newly corneal curve may affect how the tear film overlays the cornea with subsequent changing of the epithelium ocular surface leading to a dry eye; 4) in addition, osmolarity changes due to the reduced blink rate can lead to dryness. ${ }^{3-7}$

Tear meniscus is a thin strip of tear fluid with concave outer surfaces at the upper and lower lid margins. It contains most of the exposed tear volume. The absence of a tear meniscus is an indication of a dry eye. Several technologies can be used to assess the tear meniscus but have the disadvantage of being invasive. Invasive dry eye tests might cause false-positive or false-negative results. A traditional noninvasive method of measuring the tear meniscus is the slit lamp. However, it is not always easy to identify the tear meniscus. ${ }^{8,9}$

Anterior segment spectral domain optical coherence tomography (AS-SD-OCT) is a noncontact technology with a high resolution that enables the precise visualization of anterior segment structure. It is highly sensitive and specific for diagnosing dry eye and correlates well with the Schirmer's test, tear breakup time and the patient subjective symptoms. The lower tear meniscus parameters measured by AS-SD-OCT could be a more sensitive tool to evaluate postoperative dry eye than the conventional clinical tests. ${ }^{10}$ The drawbacks of using optical coherence tomography (OCT) to measure tear meniscus are the relatively high cost of the instrument and the fact that it provides information only on the quantity of tears. ${ }^{11}$

\section{Patients and methods}

This prospective randomized comparative study was performed in Ain Shams University hospitals in the period between August and December 2017. All procedures in this study adhered to the Declaration of Helsinki's ethical principles for medical research involving human subjects and were approved by the Faculty of Medicine, Ain Shams University Research Ethical Committee. A written informed consent was obtained from all participants.

\section{Inclusion and exclusion criteria}

This study included 90 eyes of 45 healthy adults. Preoperative eye refraction of all patients was between -1.75 and -9.50 diopters. They all had a stable refractive error during the previous 2 years (progressing by $<0.5$ diopters annually). The exclusion criteria included diabetes, glaucoma, systemic collagen or immune disease, corneal disease or abnormality, a history of tear replacement therapy, contact lens wear during the past year and those who used drugs that affected tear secretion and tear film stability such as corticosteroids and anti-glaucoma drugs.

In this study, there was blinding in the measurements and analysis. Tear film was measured for all patients blindly, preoperatively. The patients were distributed in a random way by a ratio of $1: 1: 1$ to the three LASIK procedures. Neither the investigator or patient had influence on treatment received. The groups were labeled as A, B and C. Each group went through a different LASIK technique. The first group of patients was subjected to the conventional microkeratome LASIK (Allegretto WaveLight EX500; Alcon Laboratories, Inc., Fort Worth, TX, USA), the second group of patients was subjected to FS-LASIK (Refractive Suite from Allegretto wave light for Femto LASIK; Alcon Laboratories, Inc.) and the third group of patients was subjected to FS-SMILE (ReLEx femtosmile; Carl Zeiss Meditec AG, Jena, Germany).

\section{Surgical methods}

All patients were treated by the same surgeon; all patients were superficially anesthetized with benoxinate hydrochloride $0.4 \%$. In the LASIK group, the corneal flap was made using the microkeratome followed by the ablation of corneal stroma. In the FS-LASIK group, the corneal flap was made with femtosecond (FS) laser followed by the ablation of corneal stroma. In the FS-SMILE group, the FS laser scanning was performed and the corneal refractive lenticule and cap-shaped micro-incision were prepared. After separating free lenticule, lenticule extraction was performed through the micro-incision. The thickness of corneal cap of 
the SMILE group was consistent with that of the LASIK and FS-LASIK groups.

\section{Postoperative management}

Patients in all groups received levofloxacin eye drops four times daily for a week, and fluorometholone $0.1 \%$ eye drops four times daily for a week, and then tapered over the following month. Carboxymethylcellulose sodium eye drops were given four times daily for 2 months after operation. Refresh plus preservative-free lubricating eye drops were used for all patients. All patients were instructed to stop the drops 3 hours prior to examination.

Postoperatively, in all patients, the tear meniscus parameters were measured after 1 week, 1 month, 3 months and 6 months by an operator who was blind to the randomization.

Measurement of the lower tear meniscus parameters was performed by AS-SD-OCT (Cirrus HD-model 5000; Carl Zeiss Meditec AG, Jena, Germany). Images were taken immediately after blinking. Scan protocol was high definition cornea. The anterior segment rastol line was applied at $90^{\circ}$ (vertical scan) at the junction between lower cornea and lower lid margin. After focusing of the instrument with a crossline (CL) centered on lower tear meniscus at 6 o'clock of the cornea, a $6 \mathrm{~mm}$ long scan was obtained. The tear meniscus height (TMH; $\mu \mathrm{m})$, the tear meniscus depth (TMD; $\mu \mathrm{m}$ ) and the tear meniscus area (TMA; $\mathrm{mm}^{2}$ ) were measured with the help of a caliber integrated in the OCT software and calculated by using the integrated analysis system available in the custom software.

Changes in the lower tear meniscus parameters were studied among each group over the different follow-up periods, and comparison was performed between the effect of the three LASIK techniques (Figures 1 and 2).

\section{Statistics}

There was also blinding in the analysis of the study. The statistician was not aware which group the results belonged to. Data were entered in an Excel sheet, edited and cleaned. Analysis of data was performed using three-factor repeated

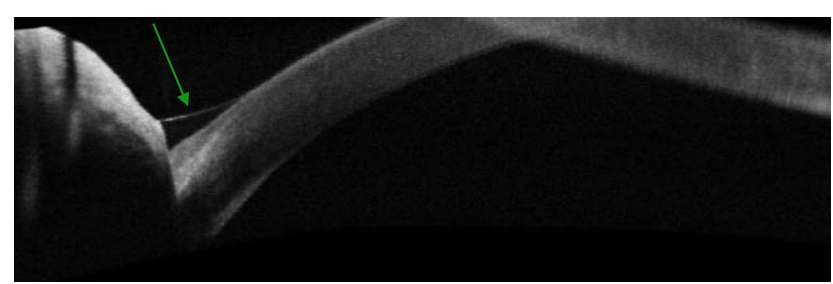

Figure I Marginal tear strip as seen by AS-SD-OCT.

Abbreviation: AS-SD-OCT, anterior segment spectral domain optical coherence tomography.

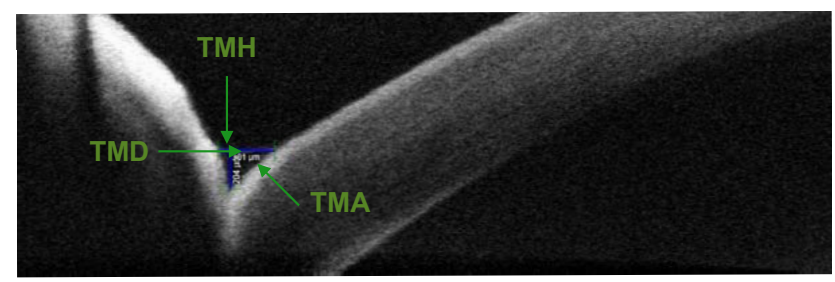

Figure 2 Tear meniscus parameters as measured by AS-SD-OCT.

Abbreviations: AS-SD-OCT, anterior segment spectral domain optical coherence tomography; TMH, tear meniscus height; TMD, tear meniscus depth; TMA, tear meniscus area.

measure analysis of variance (ANOVA) with techniques as well as between height, depth, area, follow up time and the eye. Pairwise comparisons were done using Turkey's honest significant difference test. In all tests, a $P$-value of $\leq 0.05$ was considered as statistically significant. The Statistical Package for the Social Sciences (SPSS version 24.0, IBM SPSS Statistics; IBM Corporation, Chicago, IL, USA) was used for the statistical analysis.

\section{Results}

The patients' age in this study was $31.7 \pm 7.6$ years for the microkeratome LASIK group, $31.1 \pm 8.9$ years for the FS-LASIK group and $30.0 \pm 6.1$ years for the FS-SMILE group. Patients in the microkeratome LASIK group had a spherical equivalent of refraction between -1.75 and -9.00 , those in the FS-LASIK group had a spherical equivalent of refraction between 2.25 and -9.5 , and those in the FS-SMILE group had a spherical equivalent of refraction between -3.00 and -9.25 , and all had astigmatism of $<-2.00$ diopters.

Compared with the baseline values, all tear meniscus parameters such as height, depth and area dropped after 1 week to the lowest level and started to increase over the follow-up period.

Regarding the TMH, TMD and TMA, there was a statistically significant difference between all points of follow-up and between the three techniques (Tables 1-3).

In pairwise comparison between the $\mathrm{TMH}$ in the three LASIK techniques at different follow-up points, there was a statistically significant difference between FS-SMILE and FS-LASIK in the first week $(P=0.003)$ and first month $(P=0.002)$ postoperatively. There was no statistically significant difference in TMH between both techniques after 3 and 6 months postoperatively. The FS-SMILE technique showed the least drop in TMH compared to the other techniques after 1 week, returned to lie within the $95 \%$ confidence interval (CI) of its preoperative level after 1 month and remained within the preoperative level thereafter. The FS-LASIK technique showed a larger amount of drop after 1 week and 
Table I Mean, SD and range of TMH in the different LASIK techniques at different times of follow-up

\begin{tabular}{|c|c|c|c|c|c|c|}
\hline \multirow[t]{2}{*}{ TMH } & \multicolumn{2}{|c|}{ Microkeratome LASIK } & \multicolumn{2}{|l|}{ FS-LASIK } & \multicolumn{2}{|l|}{ FS-SMILE } \\
\hline & Mean \pm SD $(\mu \mathrm{m})$ & Range $(\mu \mathrm{m})$ & Mean \pm SD $(\mu \mathrm{m})$ & Range $(\mu \mathrm{m})$ & Mean \pm SD $(\mu \mathrm{m})$ & Range $(\mu \mathrm{m})$ \\
\hline Preoperative & $0.254 \pm 0.020$ & $0.220-0.288$ & $0.255 \pm 0.032$ & $0.200-0.293$ & $0.255 \pm 0.022$ & $0.228-0.295$ \\
\hline I week postoperative & $0.167 \pm 0.027$ & $0.133-0.213$ & $0.189 \pm 0.033$ & $0.130-0.230$ & $0.224 \pm 0.019$ & $0.198-0.263$ \\
\hline I month postoperative & $0.177 \pm 0.026$ & $0.135-0.220$ & $0.198 \pm 0.034$ & $0.133-0.243$ & $0.235 \pm 0.018$ & $0.2 \mid 2-0.268$ \\
\hline 3 months postoperative & $0.193 \pm 0.029$ & $0.140-0.245$ & $0.225 \pm 0.040$ & $0.150-0.273$ & $0.244 \pm 0.019$ & $0.220-0.280$ \\
\hline 6 months postoperative & $0.217 \pm 0.029$ & $0.165-0.270$ & $0.245 \pm 0.036$ & $0.175-0.290$ & $0.25 I \pm 0.021$ & $0.225-0.288$ \\
\hline
\end{tabular}

Notes: Greenhouse-Geisser $F=324.9, P<0.001$, for the time factor; Greenhouse-Geisser $F=28.8, P<0.001$, for the time-technique interaction and Greenhouse-Geisser $F=0.258, P=0.614$, for the eye. $F$-test between techniques $=8.620$ and $P=0.001$ with significant all pairwise comparison.

Abbreviations: TMH, tear meniscus height; LASIK, laser in situ keratomileusis; FS-LASIK, femtosecond laser in situ keratomileusis; FS-SMILE, femtosecond small incision lenticule extraction; SD, standard deviation.

remained lower than its preoperative level till 6 months when it returned to the preoperative level (Table 4).

There was no statistically significant difference in TMH between FS-LASIK and microkeratome LASIK techniques after 1 week and 1 month, but there was a statistically significant difference between both techniques at 3 months $(P=0.019)$ and 6 months $(P=0.032)$ postoperatively. There was a statistically significant difference in TMH between FS-SMILE and microkeratome LASIK through all points of postoperative follow-up $(P<0.001$ and $P<0.007$; Table 4). However, LASIK showed the largest amount of drop and failed to reach the preoperative level and remained the lowest compared to other techniques at all points of time (Figure 3).

In pairwise comparison between the TMD in the three LASIK techniques at different follow-up points, there was a statistically significant difference in TMD between FS-LASIK and microkeratome LASIK at all the follow-up points. There was no statistically significant difference in TMD between FS-SMILE and FS-LASIK or microkeratome LASIK at all points of follow-up (Table 5). With FS-SMILE, TMD started to increase from the first month; with FS-LASIK, the increase started after 3 months and with both techniques, the TMD level returned to its preoperative level after 6 months. With microkeratome LASIK, TMD remained low at 6 months (Figure 4).
In pairwise comparison between the TMA in three techniques at different follow-up points, there was no statistically significant difference in TMA between FS-SMILE and FSLASIK at all points. All other pairwise comparisons between microkeratome LASIK and the other two techniques were statistically significant at all postoperative follow-up points. $P=0.001$ for FS-SMILE and $P=0.005, P=0.004, P=0.002$ and $P=0.006$ for FS-LASIK at 1 week, 1 month, 3 months and 6 months follow-up, respectively (Table 6).

With FS-SMILE and FS-LASIK techniques, TMA started to increase after the first week and attained the preoperative level at 6 months. However, with microkeratome LASIK, TMA showed the largest amount of drop and failed to reach the preoperative level till the sixth month (Figure 5).

Although the patients' mean age in this study was $31.7 \pm 7.6$ years for the LASIK group, $31.1 \pm 8.9$ years for the FS-LASIK group and $30.0 \pm 6.1$ years for the FS-SMILE group, which is considered a little bit high, there was an early significant recovery of the tear meniscus parameters in the FS-SMILE technique followed by the FS-LASIK technique. The recovery was as early as 1 month in the case of FS-SMILE.

\section{Discussion}

Corneal refractive surgery may change the stability of tear film because of injuries to nerve fibers and ocular surface

Table 2 Mean, SD and range of TMD in the different LASIK techniques at different times of follow-up

\begin{tabular}{|c|c|c|c|c|c|c|}
\hline \multirow[t]{2}{*}{ TMD } & \multicolumn{2}{|c|}{ Microkeratome LASIK } & \multicolumn{2}{|l|}{ FS-LASIK } & \multicolumn{2}{|l|}{ FS-SMILE } \\
\hline & Mean \pm SD $(\mu \mathrm{m})$ & Range $(\mu \mathrm{m})$ & Mean \pm SD $(\mu \mathrm{m})$ & Range $(\mu \mathrm{m})$ & Mean \pm SD $(\mu \mathrm{m})$ & Range $(\mu \mathrm{m})$ \\
\hline Preoperative & $0.164 \pm 0.020$ & $0.128-0.190$ & $0.183 \pm 0.017$ & $0.150-0.210$ & $0.166 \pm 0.018$ & $0.135-0.195$ \\
\hline I week postoperative & $0.136 \pm 0.021$ & $0.105-0.178$ & $0.156 \pm 0.017$ & $0.130-0.200$ & $0.145 \pm 0.018$ & $0.105-0.168$ \\
\hline I month postoperative & $0.137 \pm 0.021$ & $0.108-0.178$ & $0.162 \pm 0.017$ & $0.135-0.198$ & $0.151 \pm 0.015$ & $0.120-0.170$ \\
\hline 3 months postoperative & $0.146 \pm 0.020$ & $0.120-0.180$ & $0.172 \pm 0.026$ & $0.143-0.248$ & $0.157 \pm 0.014$ & $0.135-0.180$ \\
\hline 6 months postoperative & $0.155 \pm 0.022$ & $0.123-0.183$ & $0.177 \pm 0.017$ & $0.145-0.200$ & $0.161 \pm 0.015$ & $0.135-0.185$ \\
\hline
\end{tabular}

Notes: Greenhouse-Geisser $F=345.054, P<0.00$ I, for the time factor; Greenhouse-Geisser $F=2.168, P=0.056$, for the time-technique interaction and GreenhouseGeisser $F=0.0 .491, P=0.487$, for the eye. F-test between techniques $=6.02$ and $P=0.005$.

Abbreviations: SD, standard deviation; TMD, tear meniscus depth; LASIK, laser in situ keratomileusis; FS-LASIK, femtosecond laser in situ keratomileusis; FS-SMILE, femtosecond small incision lenticule extraction. 
Table 3 Mean, SD and range of TMA in the different LASIK techniques at different times of follow-up

\begin{tabular}{|c|c|c|c|c|c|c|}
\hline \multirow[t]{2}{*}{ TMA } & \multicolumn{2}{|c|}{ Microkeratome LASIK } & \multicolumn{2}{|l|}{ FS-LASIK } & \multicolumn{2}{|l|}{ FS-SMILE } \\
\hline & Mean \pm SD $(\mathrm{mm})$ & Range (mm) & Mean \pm SD $(\mathrm{mm})$ & Range (mm) & Mean \pm SD (mm) & Range (mm) \\
\hline Preoperative & $20.96 \pm 3.72$ & $15.25-26.00$ & $23.39 \pm 4.29$ & $15.55-28.60$ & $21.16 \pm 3.26$ & $16.50-26.80$ \\
\hline I week postoperative & $11.43 \pm 3.23$ & $7.25-18.90$ & $15.05 \pm 3.27$ & $9.40-21.50$ & $16.29 \pm 2.45$ & $11.30-21.30$ \\
\hline I month postoperative & $12.28 \pm 3.20$ & $7.50-18.65$ & $16.08 \pm 3.61$ & $9.90-21.90$ & $17.70 \pm 2.22$ & $13.45-2 \mid .30$ \\
\hline 3 months postoperative & $14.22 \pm 3.67$ & $8.35-20.20$ & $19.00 \pm 4.39$ & $12.15-25.00$ & $19.03 \pm 1.97$ & $16.10-22.40$ \\
\hline 6 months postoperative & $16.97 \pm 4.29$ & II.30-24.60 & $21.61 \pm 4.35$ & $14.3-28.25$ & $20.21 \pm 2.69$ & $16.50-25.50$ \\
\hline
\end{tabular}

Notes: Greenhouse-Geisser $F=345.05, P<0.001$, for the time factor; Greenhouse-Geisser $F=16.396, P<0.001$, for the time-technique interaction and GreenhouseGeisser $F=0.677, P=0.415$, for the eye. $F$-test between techniques $=6.536$ and $P=0.003$.

Abbreviations: SD, standard deviation; TMA, tear meniscus area; LASIK, laser in situ keratomileusis; FS-LASIK, femtosecond laser in situ keratomileusis; FS-SMILE, femtosecond small incision lenticule extraction.

epithelium. Safe and effective corneal refractive surgery correction methods for myopia are LASIK, FS-LASIK and FS-SMILE. Dry eye is considered to be the most common complication after surgery.

Mechanical microkeratome and FS-LASIK are used to create corneal flaps during LASIK surgery. Compared with microkeratome, FS-LASIK provides better predictability of flap dimensions and improves the quality of the optical surfaces. ${ }^{12}$ The FS laser is an infrared laser with a very high power. It works in the form of pulse, and can focus and separate the tissue and form plasma. Under the influence of the plasma, the FS laser may form laser blasting effect and make the tissue produce microbubbles. A large number of microbubbles are connected and produce a very precise cutting effect. ${ }^{13}$ These two factors may reduce the incidence of postoperative dry eye. ${ }^{14}$ In FS-SMILE, the flap is replaced with small FS incision and the stromal ablation is replaced with refractive lenticule removal. The optical surface is protected from a flap and from ablations. These factors are more protecting for the corneal epithelium and may further reduce the incidence of postoperative dry eye.

The AS-SD-OCT used in this study has been confirmed to be used for both quantitative evaluation of the tears and diagnosis of dry eye syndrome. It has a high sensitivity and specificity in diagnosis and has the capacity to detect early changes in tear meniscus after refractive surgery and to facilitate investigation of dry eye throughout the follow-up period after surgery. ${ }^{11,14}$ The parameters of the lower tear meniscus may better represent the total tear volume than those of the upper meniscus, and the changes in lower TMH might be a more sensitive indicator of dry eye than changes in area. ${ }^{15,16}$

In this study, we did compare the effects of LASIK, FS-LASIK and FS-SMILE on all the parameters of the lower TMH, TMD and TMA through different postoperative follow-up periods of 1 week, 1 month, 3 months and 6 months. With all three techniques, all parameters dropped after 1 week of surgery to the lowest level and started to increase in the postoperative follow-up periods. With microkeratome LASIK, TMH, TMD and TMA showed the largest amount of drop after 1 week, failed to reach the preoperative level after 6 months and remained the lowest compared to other techniques at all points of follow-up (Figures 3-5). Based on these findings, it is concluded that LASIKassociated dry eye is significantly higher in the microkeratome group during the 6-month follow-up period.

Multiple studies had been performed to study the effects of LASIK on the tear stability over different postoperative times. Yu et al ${ }^{17}$ reported that LASIK significantly altered the tear film stability and patients frequently experienced dry eye symptoms after the procedure. On the contrary, Patel et al ${ }^{18}$ reported that the average TMH and stability are not affected by LASIK, but there is considerable variability between individual eyes. Tao et $\mathrm{ll}^{19}$ showed that the upper and lower

Table $4 \mathrm{P}$-value in pairwise comparison of the TMH between the three LASIK techniques at different times of follow-up

\begin{tabular}{llll}
\hline TMH & $\begin{array}{l}\text { Microkeratome } \\
\text { LASIK vs FS-LASIK }\end{array}$ & $\begin{array}{l}\text { Microkeratome } \\
\text { LASIK vs FS-SMILE }\end{array}$ & $\begin{array}{l}\text { FS-LASIK vs } \\
\text { FS-SMILE }\end{array}$ \\
\hline Preoperative & 0.998 & 0.994 & 0.998 \\
I week postoperative & 0.075 & $<0.00 I^{*}$ & $0.003^{*}$ \\
I month postoperative & 0.093 & $<0.00 I^{*}$ & $0.002^{*}$ \\
3 months postoperative & $0.019^{*}$ & $<0.00 I^{*}$ & 0.19 I \\
6 months postoperative & $0.032^{*}$ & $0.007^{*}$ & 0.824 \\
\hline
\end{tabular}

Note: *Statistically significant difference between any two techniques at specific time of follow-up.

Abbreviations: TMH, tear meniscus height; LASIK, laser in situ keratomileusis; FS-LASIK, femtosecond laser in situ keratomileusis; FS-SMILE, femtosecond small incision lenticule extraction. 


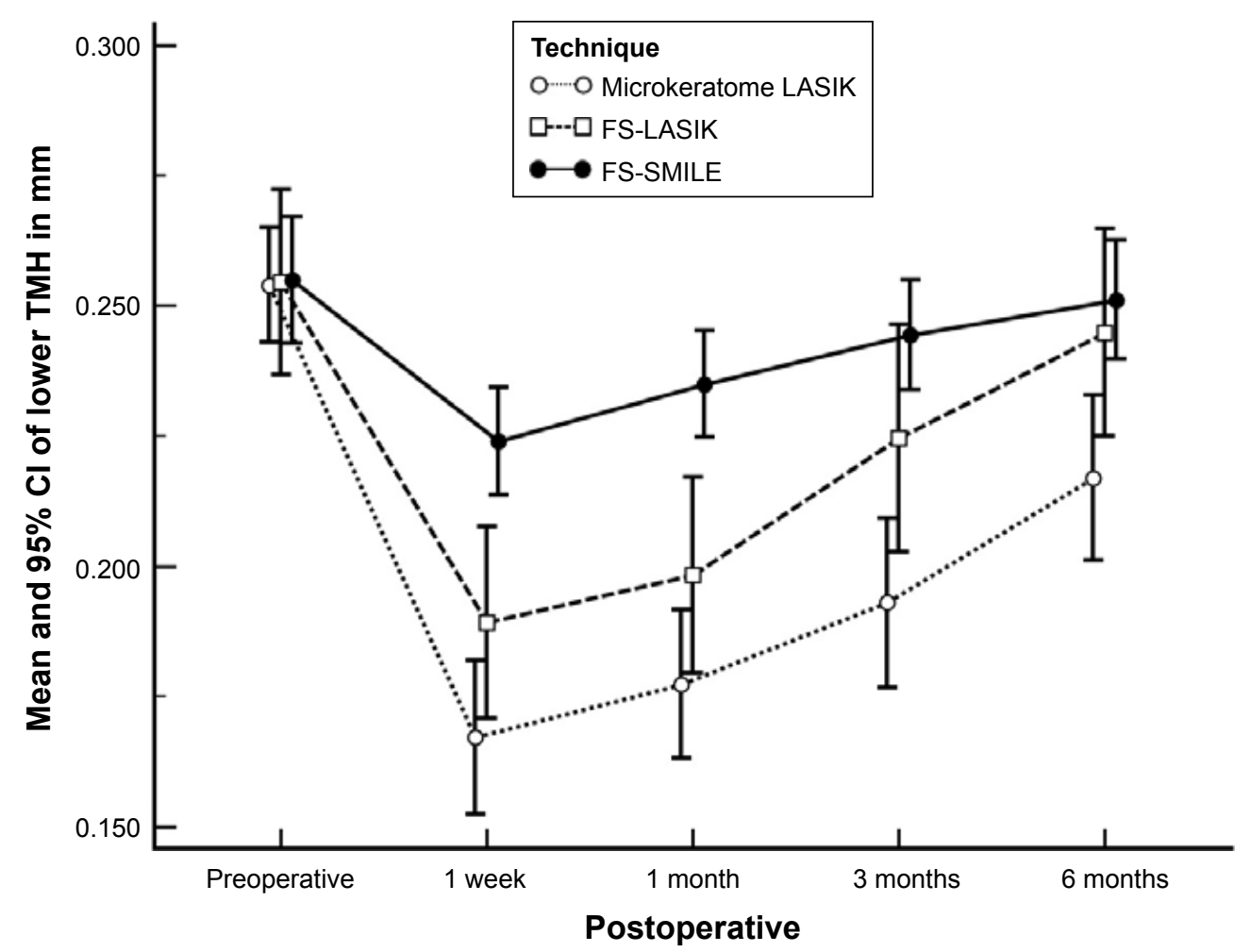

Figure 3 In all techniques, TMH dropped after I week to the lowest level.

Notes: The FS-SMILE technique showed the least drop and returned to $95 \%$ of its preoperative level after I month. FS-LASIK reached to its preoperative level at 6 months, while microkeratome LASIK did not.

Abbreviations: TMH, tear meniscus height; FS-SMILE, femtosecond small incision lenticule extraction; FS-LASIK, femtosecond laser in situ keratomileusis; LASIK, laser in situ keratomileusis; $\mathrm{Cl}$, confidence interval.

tear menisci volumes decreased 1 month postoperatively in patients who underwent LASIK and recovered to preoperative levels by 20 months.

In this study, the FS-LASIK group showed a larger amount of drop in height after 1 week and remained low till 6 months when it retained its preoperative level. TMD and TMA returned to the preoperative level by the sixth month. Although there was no statistically significant difference in TMH between LASIK and FS-LASIK after 1 week and 1 month, there was a statistical significant difference between them at 3 and 6 months (Table 4). These data indicated that the FS-LASIK may result in a lower incidence or severity of dry eye at the early stages after surgery.
In the 3-month study, Salomão et $\mathrm{al}^{7}$ found that the incidence of LASIK-associated dry eye was significantly higher in the microkeratome group than in the FS group. With the absence of significant differences between the microkeratome LASIK and FS-LASIK groups in tear menisci at 1 week, 1 month and 3 months, they concluded that the FS-LASIK may not result in a lower incidence or severity of dry eye at the early stages after surgery. Another 3-month study done by Xie et $\mathrm{al}^{20}$ showed no significant differences in the tear meniscus parameters between the microkeratome LASIK and FS-LASIK at 1 week, 1 month and 3 months, indicating that the FS-LASIK may not result in a lower incidence or severity of dry eye at the early stages after surgery. It was found that, similar to

Table $5 P$-value in pairwise comparison of the TMD between the three LASIK techniques at different times of follow-up

\begin{tabular}{llll}
\hline TMD & Microkeratome & Microkeratome & FS-LASIK vs \\
& LASIK vs FS-LASIK & LASIK vs FS-SMILE & $0.026^{*}$ \\
\hline Preoperative & $0.017^{*}$ & 0.983 & 0.288 \\
I week postoperative & $0.017^{*}$ & 0.378 & 0.216 \\
I month postoperative & $0.001^{*}$ & 0.110 & 0.128 \\
3 months postoperative & $0.004^{*}$ & 0.344 & 0.586 \\
6 months postoperative & $0.006^{*}$ & 0.066 & \\
\hline
\end{tabular}

Note: *Statistically significant difference between any two techniques at specific time of follow-up.

Abbreviations: TMD, tear meniscus depth; LASIK, laser in situ keratomileusis; FS-LASIK, femtosecond laser in situ keratomileusis; FS-SMILE, femtosecond small incision lenticule extraction. 


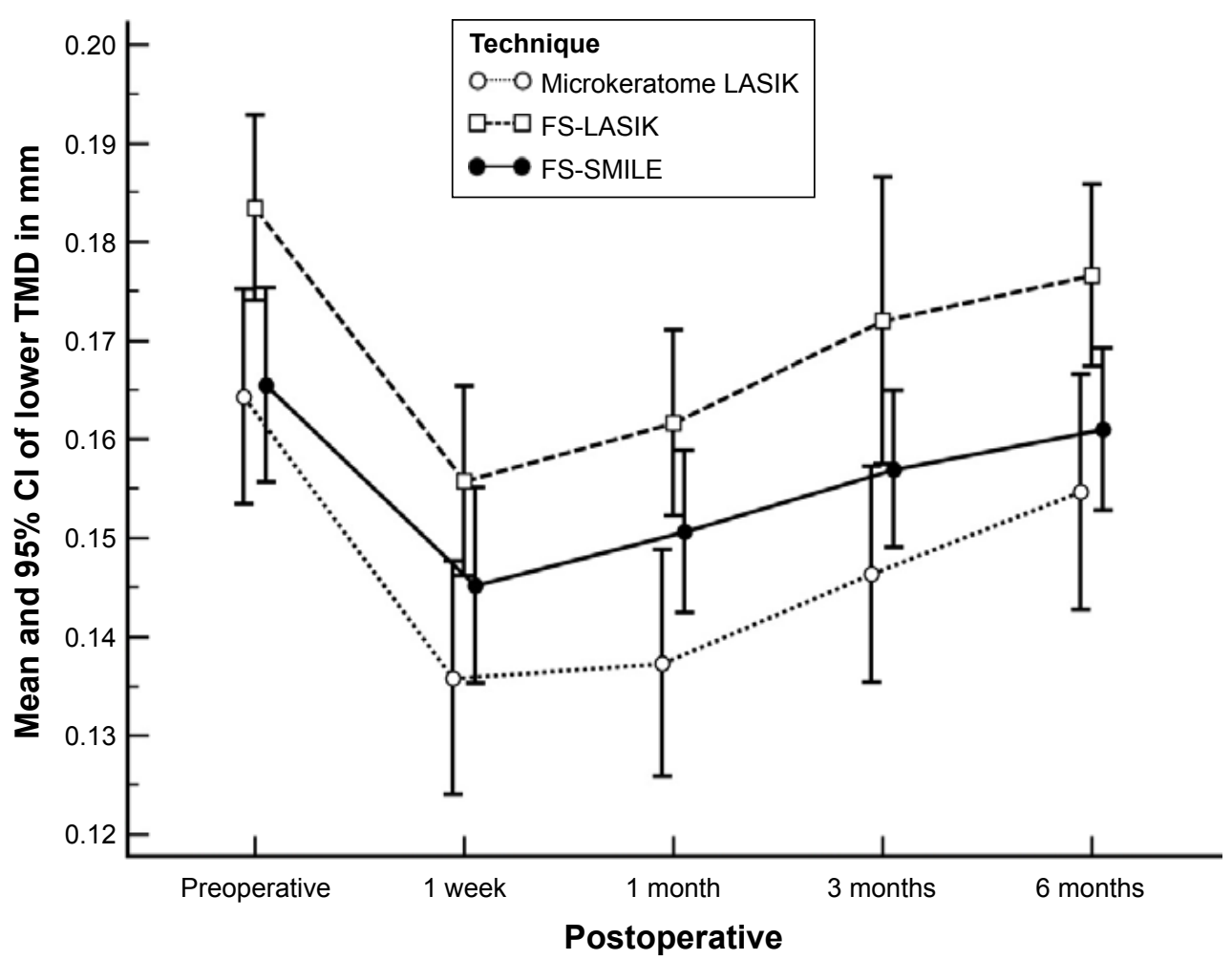

Figure 4 In all techniques, TMD dropped after I week to the lowest level.

Notes: With FS-SMILE, TMD started to increase from the first month, while with FS-LASIK, the increase started after 3 months. However, with microkeratome, TMD remained low till 6 months.

Abbreviations: TMD, tear meniscus depth; LASIK, laser in situ keratomileusis; FS-SMILE, femtosecond small incision lenticule extraction; FS-LASIK, femtosecond laser in situ keratomileusis; $\mathrm{Cl}$, confidence interval.

other studies, dry eye symptoms occur during the early stages after LASIK, peak at 1 month after surgery and later decrease gradually during the first year after the surgery. ${ }^{21-23}$

Other studies indicated that dry eye occurred after LASIK surgery with both microkeratome and FS laser. ${ }^{21,24}$ However, whether the FS laser reduces the incidence or decreases the severity of dry eye symptoms compared with the microkeratome is still controversial. ${ }^{7,24,25}$

The reason behind this controversy could be the fact that both microkeratome LASIK and FS-LASIK techniques involve many important variable factors apart from the difference in the method of flap cutting. Both techniques are used for patients with different inclusion criteria, performed by different surgeons, using a variety of different surgical and diagnostic equipment and following different follow-up protocols. The way of cutting the flap could have a different effect on the wound in some patients, and the ablation process in both techniques could be a more influential factor for eye dryness. These potential variations may result in such differences in results.

The FS-SMILE is a kind of flapless micro-resection surgery; its advantages such as small incision and light damage to the corneal nerve are conducive to rapid recovery of nervous tissue. ${ }^{10,26,27}$

In this study, the FS-SMILE technique showed the least drop in height compared to the other two techniques after 1 week. The TMH returned to lie within the $95 \%$ CI of its

Table 6 P-value in pairwise comparison of TMA between the three LASIK techniques at different times of follow-up

\begin{tabular}{lll}
\hline TMA & $\begin{array}{l}\text { Microkeratome } \\
\text { LASIK vs FS-LASIK }\end{array}$ & $\begin{array}{l}\text { Microkeratome } \\
\text { LASIK vs FS-SMILE }\end{array}$ \\
\hline Preoperative & 0.194 & 0.989 \\
I week postoperative & $0.005^{*}$ & $<0.00 I^{*}$ \\
I month postoperative & $0.004^{*}$ & $<0.00 I^{*}$ \\
3 months postoperative & $0.002^{*}$ & $<0.00 I^{*}$ \\
6 months postoperative & $0.006^{*}$ & 0.503 \\
\hline
\end{tabular}

Note: *Statistically significant difference between any two techniques at specific time of follow-up.

Abbreviations: TMA, tear meniscus area; LASIK, laser in situ keratomileusis; FS-LASIK, femtosecond laser in situ keratomileusis; FS-SMILE, femtosecond small incision lenticule extraction. 




Figure 5 In all techniques, TMA dropped after I week to the lowest level.

Note: FS-SMILE and FS-LASIK reached the preoperative level at 6 months, while microkeratome LASIK did not.

Abbreviations: TMA, tear meniscus area; FS-SMILE, femtosecond small incision lenticule extraction; FS-LASIK, femtosecond laser in situ keratomileusis; LASIK, laser in situ keratomileusis; $\mathrm{Cl}$, confidence interval.

preoperative level after 1 month and remained within the preoperative level. With FS-SMILE, both TMD and TMA reached the preoperative level at 6 months.

There was a statistically significant difference in TMH between FS-SMILE and FS-LASIK after 1 week and 1 month, but there was no statistically significant difference in TMH between FS-SMILE and FS-LASIK after 3 and 6 months postoperatively. There was no statistically significant difference in TMA between FS-LASIK and FS-SMILE at all points of follow-up time, postoperatively. The microkeratome LASIK tear parameters were the lowest. The FS-SMILE showed obvious advantage over FS-LASIK and LASIK in tear film stability, especially in recovery courses very early at 3 months postoperative.

A number of studies have demonstrated a lower reduction and faster recovery of corneal sensation after FS-SMILE than FS-LASIK. ${ }^{28-30}$

A study done by Chen et $\mathrm{al}^{28}$ to compare the postoperative long-term stability of tear film and cornea after FS-SMILE and FS-LASIK indicated that the SMILE group had better tear film stability. The SMILE group recovered faster during the 3 months after operation and were maintained stably until 12 months after operation. The FS-LASIK group demonstrated slower recovery. However, these injuries can also recover over a long term after operation.

Another study concerning dry eye after both SMILE and FS-LASIK concluded that dry eye is a transient condition that occurs in association with both techniques. SMILE does not show obvious superiority over FS-LASIK, but it may have milder subjective symptoms. ${ }^{29}$

Comparing postoperative ocular surface integrity and innervation between FS-SMILE and FS-LASIK, the SMILE procedure has fewer negative impacts on the ocular surface and corneal innervation than did FS-LASIK. FS-SMILE shows superiority over FS-LASIK by exhibiting a lower risk of postoperative dry eye. ${ }^{30}$

\section{Conclusion}

We believe that all the three techniques are safe, effective and predictable. We also think that the sooner the parameters of the lower tear meniscus, especially the height, returned to their preoperative values, the lesser the incidence of dry eye in the early postoperative period. However, dry eye symptoms are expected to occur less frequently after FS-SMILE than after FS-LASIK or microkeratome LASIK. This is because the anterior stroma is disturbed only by a small FS incision 
to extract a lenticule and there is no flap or ablation. The TMH with FS-SMILE returned almost to its preoperative level by the first month, and the TMH with FS-LASIK height started to recover from the third month to the sixth month. Microkeratome LASIK shows slower recovery.

\section{Acknowledgment}

The authors acknowledge the support of Dr Mustafa HM, Professor of Community and Public Health, Faculty of Medicine, Ain Shams University, for performing the statistics of this study.

\section{Disclosure}

The authors report no conflicts of interest in this work.

\section{References}

1. Blum M, Kunert K, Gille A, Sekundo W. LASIK for myopia using the Zeiss VisuMax femtosecond laser and MEL 80 excimer laser. $J$ Refract Surg. 2009;25(4):350-356.

2. Reinstein DZ, Archer TJ, Gobbe M. Small incision lenticule extraction (SMILE) history, fundamentals of a new refractive surgery technique and clinical outcomes. Eye and Vision. 2014;1:3.

3. Ambrosio RJ, Tervo T, Wilson SE. LASIK-associated dry eye and neurotrophic epitheliopathy: pathophysiology and strategies for prevention and treatment. J Refract Surg. 2008;24(4):396-407.

4. Shoja MR, Besharati MR. Dry eye after LASIK for myopia: incidence and risk factors. Eur J Ophthalmol. 2007;17(1):1-6.

5. Wei S, Wang Y. Comparison of corneal sensitivity between FS-LASIK and femtosecond lenticule extraction (ReLEx flex) or small-incision lenticule extraction (ReLEx smile) for myopic eyes. Graefes Arch Clin Exp Ophthalmol. 2013;251(6):1645-1654.

6. Hassan Z, Szalai E, Berta A, Modis LJ, Nemeth G. Assessment of tear osmolarity and other dry eye parameters in post-LASIK eyes. Cornea. 2013;32(7):e142-e145.

7. Salomão MQ, Ambrosio RJ, Wilson SE. Dry eye associated with laser in situ keratomileusis: mechanical microkeratome versus femtosecond laser. J Cataract Refract Surg. 2009;35(10):1756-1760.

8. Mengher LS, Bron AJ, Tonge SR, Gilbert DJ. Effect of fluorescein instillation on the pre-corneal tear film stability. Curr Eye Res. 1985; 4(1):9-12.

9. Wright JC, Meger GE. A review of the Schirmer test for tear production. Arch Ophthalmol. 1962;67:564-565.

10. Dahab A, Khafagy MM, Shaban N, Hatata R, Foaad P. Spectral-domain optical coherence tomography lower tear meniscus measurements following conventional and femtosecond laser In situ keratomileusis. 19th ESCRS Winter Meeting. Istanbul, Turkey: 2015.

11. Zhou S, Li Y, Lu AT, et al. Reproducibility of tear meniscus measurement by Fourier domain optical coherence tomography: a pilot study. Ophthalmic Surg Lasers Imaging. 2009;40(5):442-447.
12. Sarayba MA, Ignacio TS, Tran DB, Binder PSA. $60 \mathrm{kHz}$ IntraLase femtosecond laser creates a smoother LASIK stromal bed surface compared to a Zyoptix XP mechanical microkeratome in human donor eyes. J Refract Surg. 2007;23(4):331-337.

13. Lu H, Ma Q, Li C. Preliminary clinical study on femtosecond lasik surgery for the correction of myopia. Jiangxi Med J. 2011;46:450-451.

14. Durrie DS, Slade SG, Marshall J. Wavefront-guided excimer laser ablation using photorefractive keratectomy and sub-Bowman's keratomileusis: a contralateral eye study. J Refract Surg. 2008;24(1):S77-S84.

15. Vestergaard AH, Gronbech KT, Grauslund J, Ivarsen AR, Hjortdal JO. Subbasal nerve morphology, corneal sensation, and tear film evaluation after refractive femtosecond laser lenticule extraction. Graefes Arch Clin Exp Ophthalmol. 2013;251(11):2591-2600.

16. Li J, Shen M, Wang J, et al. Clinical significance of tear menisci in dry eye. Eye Contact Lens. 2012;38(3):183-187.

17. Yu EY, Leung A, Rao S, Lam DS. Effect of laser in situ keratomileusis on tear stability. Ophthalmology. 2000;107(12):2131-2135.

18. Patel S, Alió JL, Artola A, Martinez MJ. Tear volume and stability after LASIK. Refract Surg. 2007;23(3):290-298.

19. Tao A, Shen M, Wang J, Chen Q, Lu F. Tear menisci decreased 1 month after LASIK but recovered at 20 months. Eye Contact Lens. 2010;36(2): $81-85$.

20. Xie W, Zhang D, Chen J, Liu J, Yu Y, Hu L. Tear menisci after laser in situ keratomileusis with mechanical microkeratome and femtosecond laser. Invest Ophthalmol Vis Sci. 2014;55(9):5806-5812.

21. Sutton G, Lawless M, Hodge C. Laser in situ keratomileusis in 2012: a review. Clin Exp Optom. 2013;97(1):18-29.

22. Murakami Y, Manche EE. Prospective, randomized comparison of self reported postoperative dry eye and visual fluctuation in LASIK and photorefractive keratectomy. Ophthalmology. 2012;119(11):2220-2224.

23. Toda I, Asano-Kato N, Komai-Hori Y, Tsubota K. Dry eye after laser in situ keratomileusis. Am J Ophthalmol. 2001;132:1-7.

24. Farjo AA, Sugar A, Schallhorn SC, et al. Femtosecond lasers for LASIK flap creation: a report by the American Academy of Ophthalmology. Ophthalmology. 2013;120(3):e5-e20.

25. Golas L, Manche EE. Dry eye after laser in situ keratomileusis with femtosecond laser and mechanical keratome. J Cataract Refract Surg. 2011;37(8):1476-1480.

26. Zhang Y, Jia B, Zhang Y. Analysis on tear film after LASIK by femtosecond laser with Oculus corneal topography. Int Eye Sci. 2014;14: $1116-1118$.

27. Sun CC, Chang CK, Ma DH, et al. Dry eye after LASIK with a femtosecond laser or a mechanical microkeratome. Optom Vis Sci. 2013;90(10):1048-1056.

28. Chen D, Yu K, Zhang X, Liao H. Comparison of postoperative long term stabilities of tear film and cornea after femtosecond small incision lenticule extraction and femtosecond laser in situ keratomileusis. Biomed Res. 2017;28(15):6662-6666.

29. Shen Z, Zhu Y, Song X, Yan J, Yao K. Dry eye after small incision lenticule extraction (SMILE) versus femtosecond laser-assisted in situ keratomileusis (FS-LASIK) for myopia: a meta-analysis. PLoS One. 2016;11(12): e0168081.

30. Kobashi H, Kamiya K, Shimizu K. Dry eye after small incision lenticule extraction and femtosecond laser-assisted LASIK: meta-analysis. Cornea. 2017;36(1):85-91.
Clinical Ophthalmology

\section{Publish your work in this journal}

Clinical Ophthalmology is an international, peer-reviewed journal covering all subspecialties within ophthalmology. Key topics include: Optometry; Visual science; Pharmacology and drug therapy in eye diseases; Basic Sciences; Primary and Secondary eye care; Patient Safety and Quality of Care Improvements. This journal is indexed on Submit your manuscript here: http://www.dovepress.com/clinical-ophthalmology-journal

\section{Dovepress}

PubMed Central and CAS, and is the official journal of The Society of Clinical Ophthalmology (SCO). The manuscript management system is completely online and includes a very quick and fair peer-review system, which is all easy to use. Visit http://www.dovepress.com/ testimonials.php to read real quotes from published authors. 